\title{
Best Practices for Training the Structures Flight Test Engineer
}

\author{
Wendy N. Hashii ${ }^{1}$ \\ United States Air Force Test Center (412 Test Wing), Edwards Air Force Base, CA, 93524, USA \\ and \\ Randolph C. Thompson ${ }^{2}$ \\ NASA Armstrong Flight Research Center, Edwards Air Force Base, CA, 93524, USA
}

\begin{abstract}
The Structures Flight from $4^{\text {th }}$ Test Wing and the Aerostructures Branch at NASA Armstrong Flight Research Center at Edwards Air Force Base have developed a set of best practices for training a structures flight test engineer. These practices represent the hard-won lessons learned and best practices from training generations of engineers to perform high risk envelope expansion and developmental flight test. Collectively, these organizations have tested many of the world's most advanced and innovative aircraft, including the B-2, C-17, F-22, F35, RQ-4, X-29, F-18 High Angle of Attack Research Vehicle, X-53, G-III Adaptive Compliant Trailing Edge, and X-56.
\end{abstract}

\section{Introduction}

$\boldsymbol{N}^{\mathrm{s}}$ AFE and effective (i.e., successful) flight testing can be described as so many parts preparation, so many parts skillful execution, and so many parts luck." The goal is to drive any necessity for luck to zero by increasing preparation and skillful execution. A comprehensive general training plan, as well as a discipline-specific training plan, impacts both preparation and execution, and is a foundation for successful flight testing. This is particularly true for high-risk envelope expansion testing common to structures developmental and research flight test.

"Aviation in itself is not inherently dangerous. But..., it is terribly unforgiving of any carelessness, incapacity or neglect." " Entering flight-test with untrained or inadequately trained engineering staff can be just as "unforgiving" as an untrained or inadequately trained pilot. In fact, entering a flight test program with untrained or inadequately trained engineers leaves the engineers unable to perform their safety duties (incapacity) and is careless and neglectful. This is recognized by organizations around the world as schools have been developed for the express purpose of training individuals in the discipline of flight-test (the United States Air Force Test Pilot School, the United States Naval Test Pilot School, Empire Test Pilots' School, the École du Personnel Navigant d'Essais et de Réception, and others). However, these test pilot schools generally train engineers to become test conductors/directors, and do not provide sufficient depth within a single engineering discipline to train a discipline engineer.

Experience has shown that standardized training plans, which can be tailored to each test program, provide test engineers who are prepared to meet the challenges of high-risk developmental and research flight test. The purpose of this paper is to present the experience and best practices (of the United States Air Force (USAF) 412 Test Wing (TW) and the National Aeronautics and Space Administration (NASA) Armstrong Flight Research Center (AFRC)) for training structures flight test engineers. The decades-long experience of these organizations have led to the development of successful training methodologies for high risk developmental and research flight test programs. For the purposes of this paper, a structures engineer is defined as someone engaged in testing aircraft structures within the sub-disciplines of flutter, loads, or vibro-acoustics.

\footnotetext{
${ }^{1}$ Structures Technical Expert, 412 Test Wing, Air Force Test Center, AIAA member.

${ }^{2}$ Aerostructures Branch Chief, NASA Armstrong Flight Research Center, AIAA Member.

* This is adapted from a well-known quote by David L. Baker. "Flying is so many parts skill, so many parts planning, so many parts maintenance, and so many parts luck. The trick is reduce the luck by increasing the others." The authors were unable to find the original reference of this quote.

$\dagger$ This quote is attributed to Captain A. G. Lamplugh of the British Aviation Insurance Group, London, circa the early 1930s.
} 


\section{An Overview of Basic Structures Engineering Training}

A formal training plan aids in providing organizations and individuals a training framework and aids engineers on their training journey. Unfortunately, there is a temptation to consider a training plan a checklist which, once completed, means the engineer is fully prepared for any test program to which they are assigned. This is naïve and increases risk to a test program by (potentially) supplying it with educated, but untrained, individuals. An ideal training plan will emphasize attaining knowledge and experience (over completing a checklist) and will include three basic areas: formal education, supplemental education, and on-the-job or experiential training. Figure 1 depicts the three types of training, progressing from formal (passive learning) to more experiential (hand-on learning). As shown in the figure, experiential training is the most significant and provides the foundation of a qualified structures engineer.

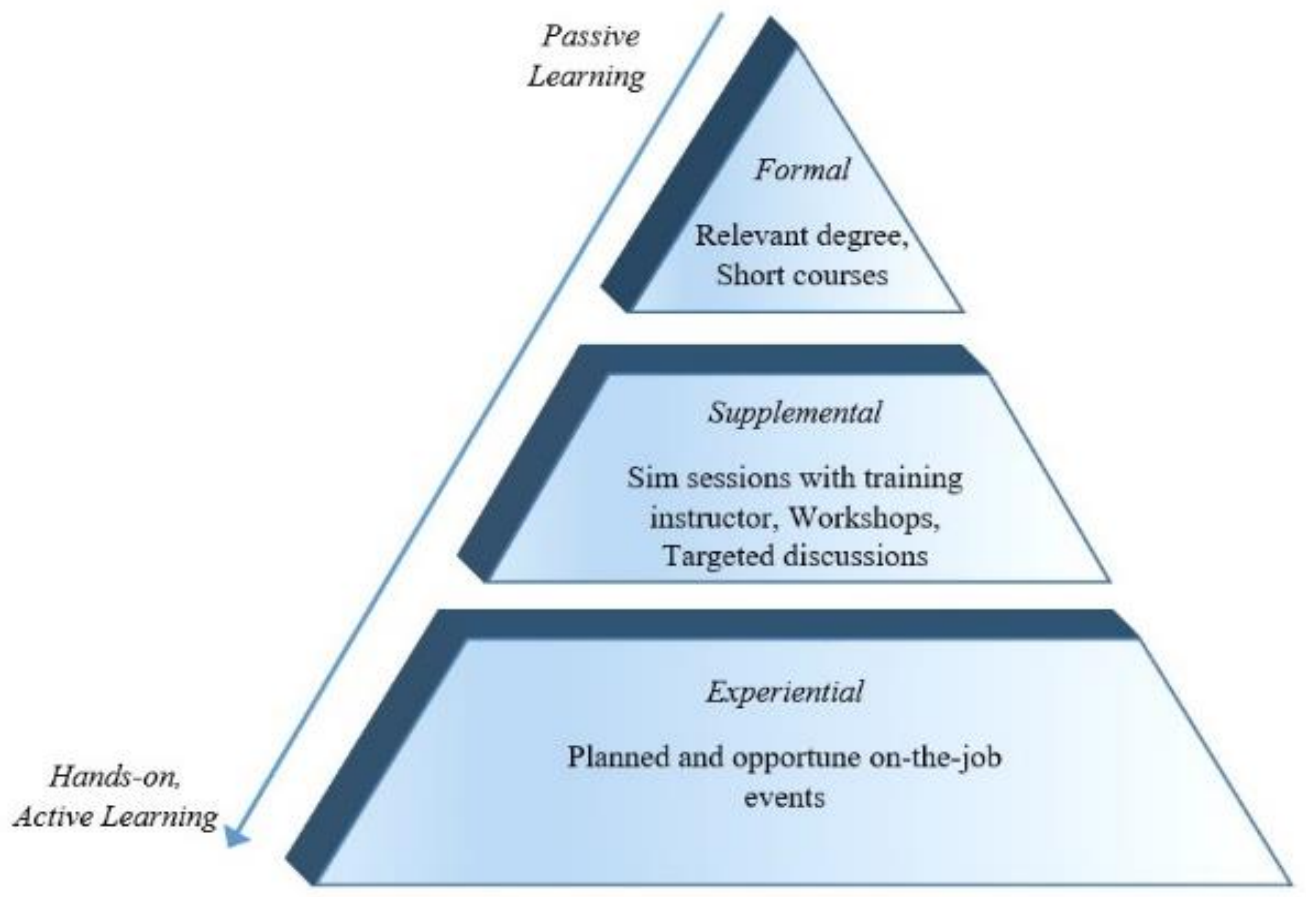

Fig. 1 Top-level depiction of training modalitites, as adapted from the National Training Laboratory's learning pyramid

Engineers beginning a career in structures flight test, either newly graduated or transferring from another discipline, require a sound educational background in basic engineering principles. Ideally, the engineer will have a Bachelor of Science (B.S.) degree (or higher) in a relevant discipline (aerospace or aeronautical engineering, mechanical engineering, physics, etc.). Most helpful are courses of study that include the following topics: structural mechanics, composite structures, structural dynamics, aeroelasticity, aerodynamics, flight mechanics, control system design and analysis, and instrumentation.

Formal education may also include short courses in topics that were either not available or not taken during the B.S. course of study, including many of the subjects previously mentioned. In addition, short courses in various finite element analysis (FEA) tools (such as MSC/NASTRAN ${ }^{\odot}$ ), aeroelasticity analysis tools (for example, ZONA's ZAERO $^{\odot}$ ), and a control system software package (like $\mathrm{Matlab}^{\odot}$ or Python ${ }^{\odot}$ ) would aid the engineer in learning the tools of the trade. Even in organizations where engineers do not directly perform these types of analysis, these courses allow the engineer to understand the assumptions and applicability of the analysis that forms the basis of an initial airworthiness certification and preliminary pre-flight predictions. Training in control room data visualization software (like Symvionics' IADS ${ }^{\odot}$ ) can be useful. Ensuring that test engineers understand the flow of data from the initial measurement on the aircraft to when it is displayed on a control room screen is critical for ensuring the quality and validity of test data. As a result, courses in transducer and instrumentation systems, digital signal processing, statistics, and design of experiments can be critically important. Organizational training in crew resource management (CRM), and test and safety planning generally round out formal training.

Supplemental education consists of a bridge (or interim layer) between formal education and experiential training. This type of training may include formal training events such as simulated control room sessions, workshops, technical 
interchange meetings with sister organizations, and lessons learned (or postmortem) discussions. These types of training events serve to create a knowledge-based organizational culture with a strong focus on safety.

Experiential training is the most critical training, and provides the foundation of a well-qualified structures flight test engineer. It is through this training that the engineer begins to learn the structures discipline aspects of flight test. The training plan should accommodate planned as well as opportune events that build the engineer's experience base. There may be a time when an opportune experience may necessitate the need to delay other work knowing that the experience will pay dividends later. These experiences begin well before the first ground or flight test. A structures flight test engineer who is involved in all phases of the developmental program (as shown in Fig. 2) will have a deeper and more comprehensive understanding than one who is present merely for test execution. Each milestone provides an opportunity to learn about the system under test. Additionally, a test engineer will have valuable input into early decisions that impact subsequent test and evaluation. (Does the test strategy align with the acquisition strategy? Are the key performance parameters measurable/testable? Is the design of the instrumentation system sufficient? Is the design of flight test aids sufficient?)

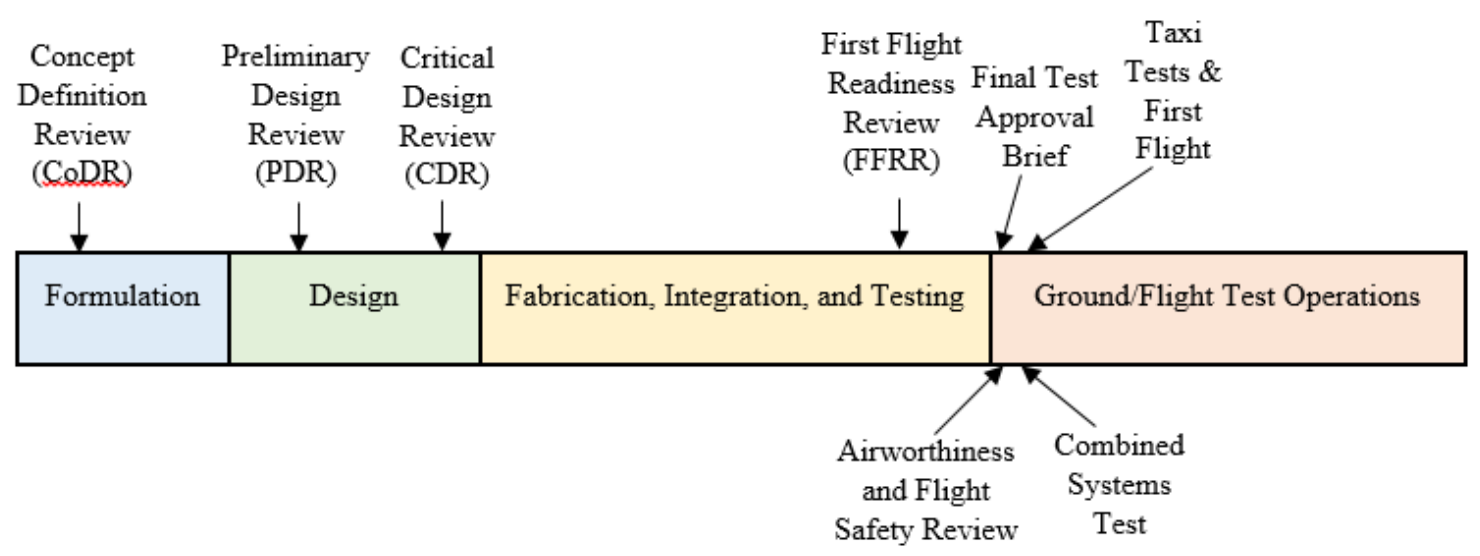

Fig. 2 AFRC project phases

Naturally, a significant part of experiential training for a structures flight test engineer includes control room monitoring of flight test missions. The purpose of control room monitoring of developmental or research aircraft during high risk testing is to mitigate test hazards. This is done through gained insight into vehicle performance by either ensuring that safety of test limits are maintained or by providing the ability to develop real-time predictions of system performance for the next test point in the test progression.

Additional discussion on selected training topics is contained in the following sections.

\section{Mission Control Room (MCR) Support Training}

When most people think of flight test training, the first thing that probably comes to mind is mission control room training. However, as shown in Fig. 3, most training and preparation either occurs prior to setting foot in the control room, or after leaving the control room (post-test).

Long lead training is accomplished as general preparation to become a qualified control room engineer. The simulation sessions are of particular note. Ideally, these sessions are held with a full control room team in a representative mission control room with a remote link to an aircraft simulator flown by the mission pilot. Traditionally, these types of simulations have not included realistic airframe structural responses. However, recent advances in modeling and prediction techniques should allow future simulations to include realistic structural responses.

The pre-flight training and preparation usually occurs within days (or hours) of a particular test mission. This preparation is mission specific. The mission rehearsal simulations have been found to be particularly useful in preparing a test team for a high risk structures flight test mission. These sessions allow the structures engineer to see how the pilot will fly the maneuver in practice (versus a predicted specification maneuver) and to provide the pilot with feedback on maneuver execution. For utilitarian purposes, the mission rehearsal simulation should be performed with the pilot and engineers who planning to execute the mission. 


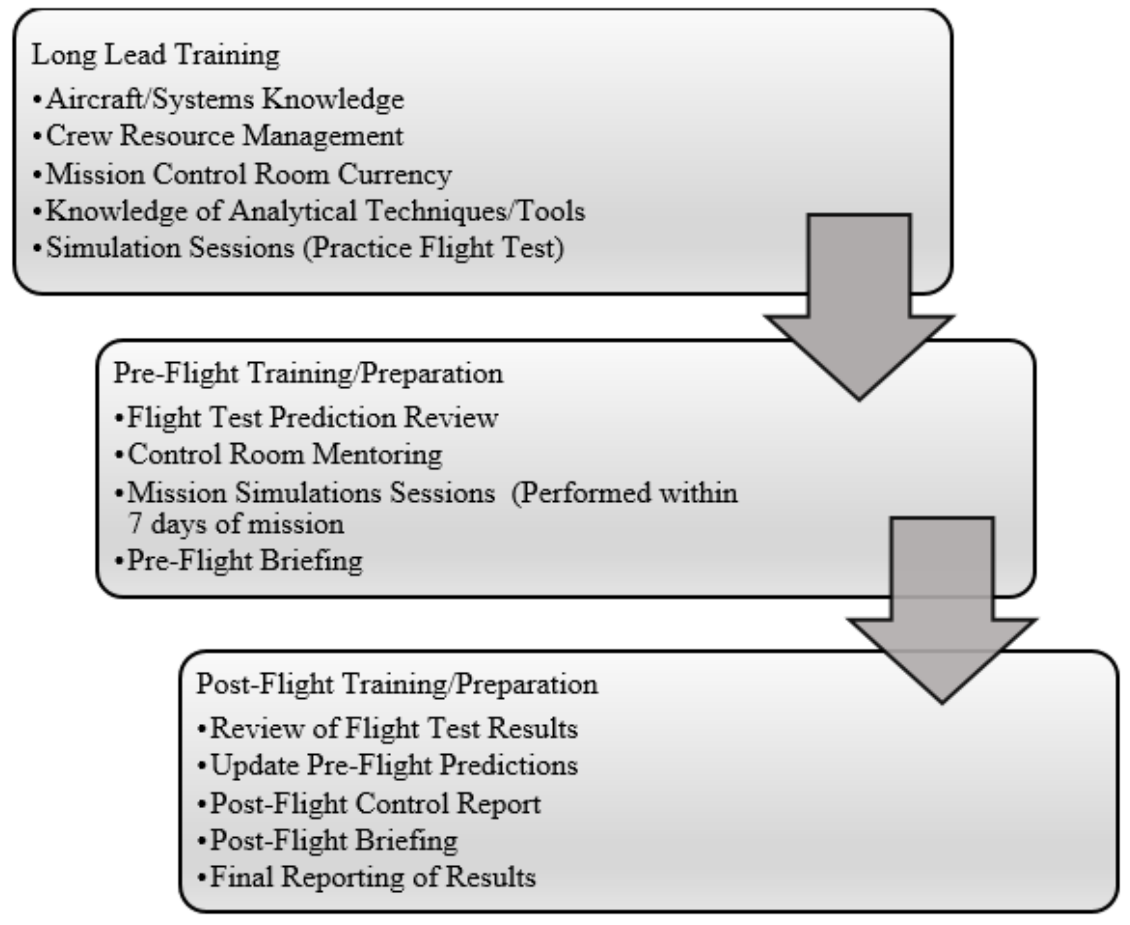

Fig. 3 Components of mission control room training

Post-flight training and preparation can occur either immediately following a mission (as for a post-flight briefing) or months later (in reporting results). The final reporting of results is generally viewed as a way to communicate flight test results up a leadership chain or to a customer. However, a final flight test report is arguably one of the most valuable documents that exists for training future engineers. A well-written report that documents not only test results but also data analysis techniques, significant findings, and lessons learned is a treasure trove of information for any engineer embarking on a new flight test program. However, all too often, these reports are seen as just a summary of final results with very few resources invested in producing a quality product. Or, worse, they have been regarded as unnecessary since final structural clearances are usually produced through a correlation of flight test results with structural analysis (leading to a final structural analysis report).

\section{A. Simulation Sessions (Practice Flight Test)}

One consistent lesson learned from project to project has been the value of a flight test support simulator that is co-located with the flight test team [Ref. 1]. Structures tests (in particular, loads and buffet) often have maneuvers that are difficult to perform, and test conditions that are difficult to achieve. A local flight test support simulator allows the test team to practice maneuvers, and to experiment with different setup techniques in order to achieve the desired test conditions. A simulator can also be used to train new structures flight test engineers, or to maintain currency for experienced engineers. In particular, a simulator can be used to tailor a training scenario for a specific purpose (e.g., preparation for low altitude buffet maneuvers with negative specific excess power). Having the capability to practice test execution (either for use in test plan development, or in preparation for mission support) has substantial dividends in both test efficiency and test safety.

In order to be an effective training device, the simulator should include a high-fidelity, up-to-date software model of the flight controls, aerodynamics, and propulsion systems. Additionally, the simulator hardware should include realistic displays and a representative control stick (such that the feel of the stick is similar to the actual aircraft). However, full dome projection systems, motion-based simulators, or simulators that include flight control hardware (for the actuators and/or hydraulic systems) are not required. The control room displays should be identical to the displays that are used in the actual flight test control room. It is generally beneficial to have the control room displays co-located with the simulator, as shown in Fig. 4. Whereas some training scenarios have stood up an actual control with a link that fed data from the simulator into the control room, this setup can be substantially more expensive to run. Test teams that have not had control room displays co-located with the simulator have tended to significantly underutilize the simulator as a result of the expense of standing up a control room. 


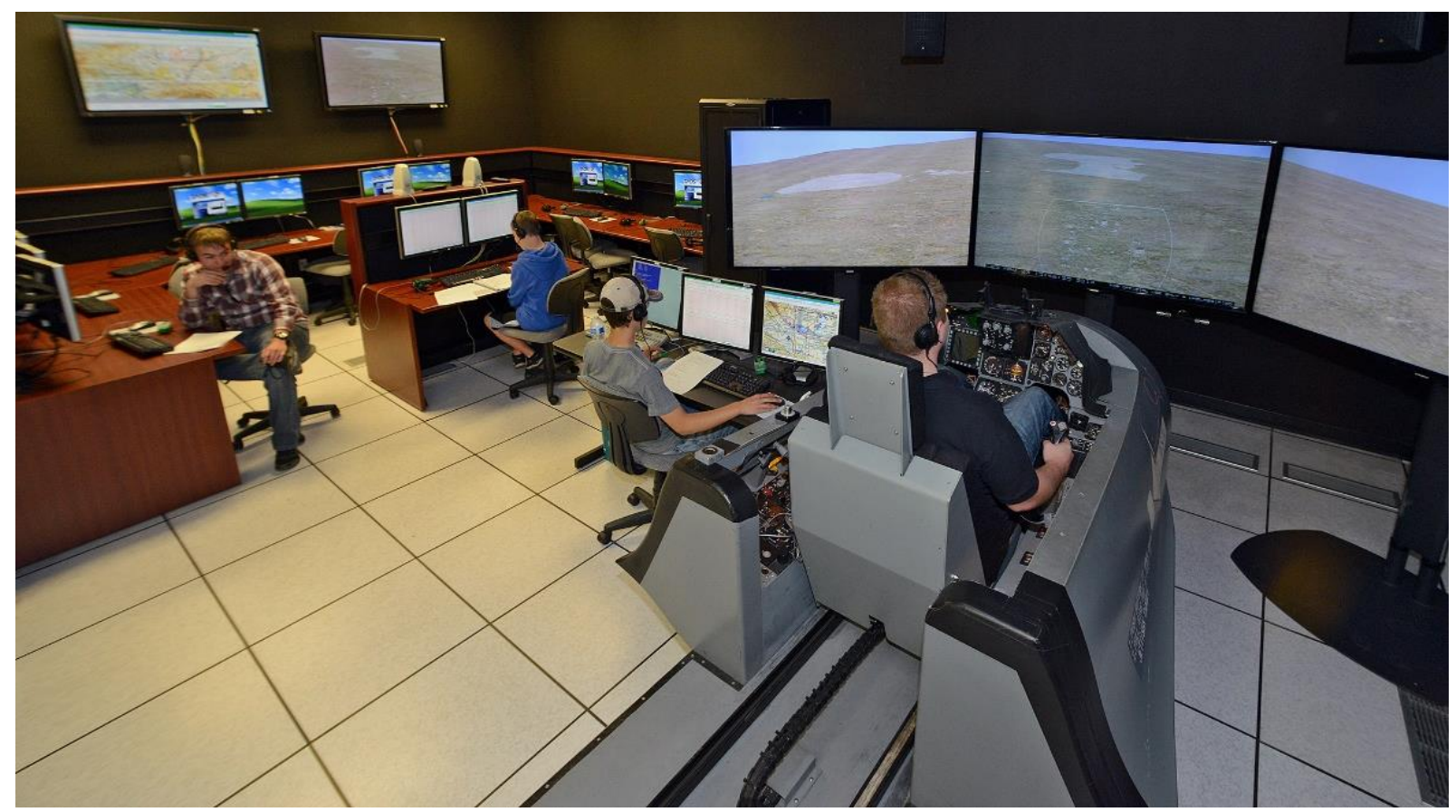

Fig. 4 Mission control room training at the Integration Facility for Avionics System Testing (IFAST) at Edwards Air Force Base

\section{B. Control Room Special Qualifications}

A standard control room qualification plan generally consists of a review of local procedures or operating instructions, a basic class in CRM principles, a discussion of control room procedures (terminology, discipline, operation of the communications console, security procedures, etc.), a briefing covering basic aircraft or systems knowledge (operating limits, emergency procedures, etc.), and a minimum number of mentored flights. High risk testing (to include loads and flutter) may also include a special qualification that is designed to familiarize a test team with a specific type of test. This special qualification may consist of a briefing of specific test procedures, simulatorbased training scenarios, and a mentored (loads or flutter) flight.

Some test teams have foregone any special qualification requirements, and have merely required a control room engineer to have participated in any control room mission (regardless of mission type) in order to become control room qualified. This practice is particularly prevalent for projects with a smaller scope where relevant test missions may be fewer in number, and it can be difficult to meet training requirements that entail a certain number of mentored flights. In this case, relevant control room training scenarios conducted with a flight test support simulator would have been preferred over control room support of a non-relevant mission. A good, simulated training scenario can replicate the control room terminology and discipline while also providing a relevant mission support example (that includes control room screens and maneuver types). In contrast, a structures engineer supporting an avionics mission to train for a loads test flight may obtain a good view of an actual control room, but will receive little relevant experience that is necessary to support an actual loads mission.

\section{Special Qualifications for Test Pilots}

Pilot training is often not considered when discussing discipline specific training. However, although it is not technically a part of MCR training, it is a crucial component of mission execution that should not be overlooked in any new flight test program. Generally, discipline specific pilot training is centered in four different areas: test pilot schools (TPS), special qualifications, pilot proficiency, and pre-flight mission simulations.

A TPS curriculum provides the first introduction to structures flight testing for most test pilots. As a result, TPS provides a foundational experience for most test pilots. However, due to time constraints (given the extensive amount of course material that must be covered within the course timeline), a TPS generally does not provide the depth of experience required for a test pilot to become truly proficient at structures flight testing nor can it provide the level of detail necessary for a pilot to become familiar with a particular airframe. Therefore, each test organization must develop its own special qualification requirements for high risk testing. For example, there might be special 
qualification requirements for both loads and flutter testing (both types of tests are generally considered high risk). These special qualifications might include:

- detailed briefing on the characteristics of the system under test by a discipline engineer,

- briefings or demonstrations of special equipment (like flight test aids or a flutter excitation system),

- practice simulation sessions attended by a discipline engineer and/or an experienced structures test pilot, and

- practice flights (with representative challenging maneuvers) monitored by a discipline engineer and an experienced structures test pilot (either in the control room or as a chase pilot).

At the beginning of most flight test programs (before anyone at the test organization has any real experience with structures testing), there is a general belief that any test pilot can perform any test mission, and there is great resistance to establishing a cadre of pilots that specialize in structures testing. The special qualifications can help overcome this initial belief, and as pilots gain more experience they generally become some of the greatest advocates for this type of training.

Pilot proficiency is directly related to safety (not just efficiency). For example, an experienced loads pilot who can execute a loads mission with fewer repeats (due to being off condition or having an incorrect inceptor position, for example) has less risk of inadvertent overloads than an inexperienced pilot who requires multiple repeats to execute a required maneuver. In particular, an excessive number of repeat maneuvers can result in pilot fatigue and frustration, which leads to an even greater risk of inadvertent overloads.

Finally, a pre-flight simulation session (performed within a reasonable timeframe of the test mission) can allow a pilot to practice difficult-to-achieve maneuvers and can give control room engineers an opportunity to provide feedback to the pilot. It can also provide valuable insight into how the pilot will fly the mission and the expected aircraft response to actual pilot inputs (versus the perfect specification maneuvers that are the basis of most pre-flight analytical predictions).

\section{Additional Supplemental Training}

The training discussed in this section can supplement both experiential training and formal education. These types of training events are designed to provide a framework to build a learning environment where education and training occurs as a fundamental part of the organizational culture.

\section{A. Lessons Learned}

Documenting lessons learned is one of the most important tasks that a test organization can perform. However, during test execution, there is little effort given to documenting lessons learned. With the majority of the effort spent executing tests, performing analysis, updating test and safety packages, and writing technical reports, there is little time left to document lessons learned as they happen. Although there is some level of effort devoted to documenting lessons learned at the completion of a project, this after-the-fact process is rarely rigorous. In particular, for large test programs, there can be a significant turnover of key personnel from the first flight to the last flight. As a result, many early lessons are often lost or forgotten.

Periodic group discussions (or post mortems) can help to ensure that significant lessons learned are documented throughout the life of a project. For example, these discussions might be scheduled to occur following a major event or at planned "stop and consider" points (e.g. after completing 80\% Design Loads Limit (DLL) testing and prior to proceeding to $100 \%$ DLL testing). A series of questions may be proposed to the test team to help instigate the discussion. For example,

- What went well on the project?

- What did not go well?

- What was the single most frustrating part of the project?

- Were the test objectives clear?

- Were you properly provisioned to execute the test? Did you have enough personnel? Did you have the appropriate tools?

- Was the schedule realistic?

- Was the test planning sufficient?

- Was the safety planning sufficient?

- What would change about our process?

- If you had this to do all over again, what would you do differently next time?

In order to keep the discussion focused on lessons learned (as opposed to finger pointing), it can be helpful to have someone moderate the discussion who is familiar with the project but who is not emotionally invested in the project. 
Documenting the discussion with written minutes can help subsequent test teams implement the lessons learned to prevent repetition of the same error. Additionally, creating an event-based questionnaire that can be given to test teams can help a test team focus on the questions they should be working to resolve in order to avoid repeating mistakes.

\section{B. Building Experience across Organizations}

Cross organizational training can be an excellent way to gain experience that may otherwise be lacking. For example, ground tests that do not occur very often (e.g., a strain gauge calibration or static) can provide a valuable experience for an engineer. However, it can be difficult to obtain this experience with a test that occurs infrequently. Other, related organizations can often help to alleviate this experience gap. An engineer exchange can allow engineers to gain experience with tests or analysis methodologies that they would not receive in their home organization. Often, the most vigorous opposition to this type of exchange comes from managers who are unwilling to release anyone from their organization based on the reasoning that "we are too busy." This is shortsighted and deprives the organization of invaluable training opportunities that will pay enormous dividends later.

In addition, a close working relationship among related organizations can allow for sharing of resources, personnel, information, and expertise. This type of symbiotic relationship has allowed short term personnel deficits to be fulfilled by engineers from another organization, allowing both organizations to benefit. One organization was able to fill a temporary personnel shortfall while the other organization was able to gain experience that they would not have otherwise received.

\section{Tailored Training}

Historically, short courses have been used to fulfill specific gaps in formal education or to provide refresher training. Although this type of training can be very valuable, it can be difficult for engineers to directly apply the theories discussed in the class to real-world applications. Additionally, if the theories or applications discussed in the class are not applied within a relatively quick period of time, they can be quickly forgotten.

Tailored training that is designed to fulfill a specific need (i.e., thermal modeling for hypersonic applications) and is available on demand can provide engineers with specific training opportunities as the need arises. This allows engineers to directly apply what they have learned for better retention. Additionally, training that is provided by subject matter experts within the organization (for example, NASA's Engineering and Safety Center Academy, as shown in Fig. 5) can contextualize the training to suit the specific needs of the organization. It can be difficult for an outside organization to provide a similar level of training on demand.

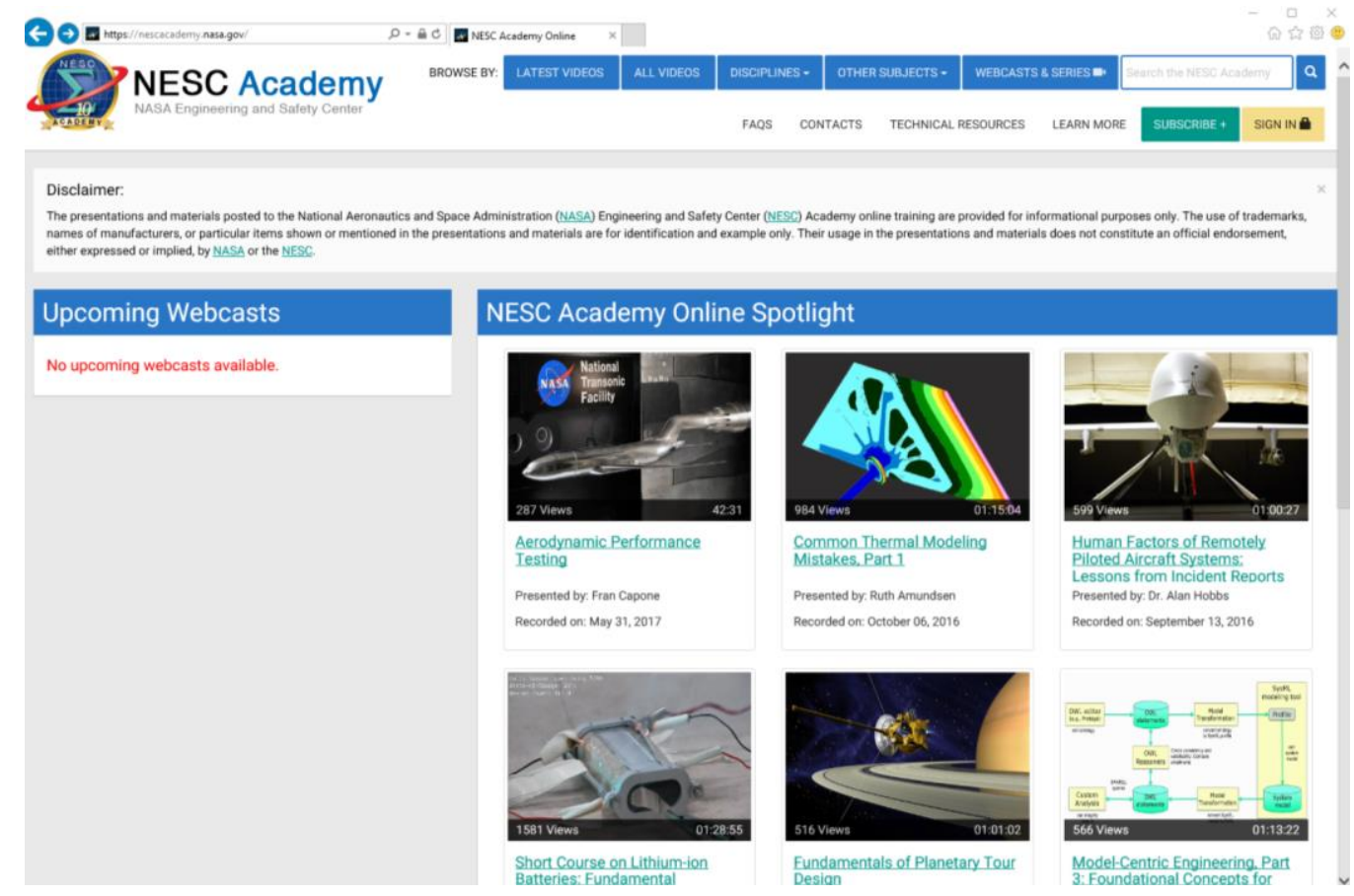

Fig. 5 NASA Engineering and Safety Center Academy 


\section{Special Considerations}

There are many circumstances that arise during a flight test program that do not fit neatly into one of the above categories that nonetheless can have a large effect on training (and safety) and, therefore, deserve special consideration.

\section{A. High-Paced Versus Low-Paced Flight Test Program}

When conducting a high-paced (generally large) flight test program, there are often multiple teams of engineers working together to clear an envelope with multiple sorties per week (or per day). Occasionally, engineers from other locations may be called in to support flight test on a temporary (or short term) assignment. As a result, effective team communication becomes imperative as each engineer must be familiar with the results from a mission with which he or she may not have been directly involved. Additionally, due to the high pace of the program, it can be difficult to keep up with data analysis of flight test results. In this type of environment, the following have found to be beneficial: daily team meetings, detailed post-flight reports emailed to all team members, dedicated team assigned responsibility for a given component (e.g., wing loads), and defined break points where flight test analysis must be completed prior to continuing testing.

Conversely, a low-paced (usually small) flight test program must be concerned with getting a control room team trained to operate in a control room environment without any recent control room experience and maintaining currency with a sporadic flight schedule. In this scenario, practice flights or simulation sessions can be invaluable in getting a test team up to speed and in maintaining currency. Additionally, allowing test team members to support other projects (or even other test organizations) on a temporary basis would provide some necessary breadth and depth of experience.

\section{B. The Value of Technical Expertise}

There is often a perception that any engineer can support any discipline with a minimal amount of additional training. This perception undervalues the level of technical depth and detail that are required to safely conduct a flight test program. A good, experienced structures flight test engineer will have detailed technical knowledge of the system under test as well as a deep understanding of structural analysis methods. However, a flight test engineer must also understand aerodynamics, flight mechanics, test techniques (for both flight and ground testing), instrumentation and digital signal processing, statistical methods, etc. This level of knowledge is not easily gained over only two to three years. But, those who have achieved expertise within the discipline of flight test structures engineering not only provides invaluable technical support (in terms of analyzing data, researching and developing new tools and techniques, monitoring safety of test parameters, analyzing data, briefing results/issues to managers/customers, etc.) but also trains and develops the next generation of engineers.

As a corollary, flight test managers must understand the discipline that they lead. Simply having good managerial skills should not imply that one would be a good manager of any given technical discipline. Studies have shown that having a technically competent boss has a more significant impact on employee satisfaction than salary [Ref. 2]. These types of studies have generally focused on results such as employee morale and retention. However, when the "business" is flight test, employee morale and retention directly impact safety.

\section{First Flight Training versus Subsequent Flight Training}

Generally, first flight training is some of the most rigorous flight test training that occurs. For good reason, a lot of resources are expended on training a first flight team. For aircraft systems testing (electrical, hydraulic, flight controls), the first flight is arguably the most risky. However, for structures, first flight is generally the least risky flight. It is usually performed within a small envelope that has been cleared via structural analysis to have sufficient margin that structural monitoring is usually not required. However, a structures engineer may participate in first flight activities to checkout screens and instrumentation, and to get a first look at monitored data.

For structures testing (primarily loads and flutter), the risk level tends to increase as testing progresses and you reach the edges of the envelope where there is less margin and maneuvers are often more difficult to complete (at high speeds, high load factors, low altitudes, etc.). Additionally, with a large test program, there can be significant turnover between the first flight team and the team conducting testing at the edges of the envelope. However, there tends to be a large discrepancy in both the quantity and quality of training provided to the first flight test team versus subsequent members of the test team. For example, an engineer who is part of the first flight test team may be required to attend multiple practice simulation sessions with a full control room that is linked to a piloted simulation, detailed aircraft familiarization briefings given by aircraft design engineers, and several team planning meetings (first flight readiness reviews). Conversely, an engineer who comes on-board later in the flight test program may receive a couple of 
mentored flights (that may or may not be structures test flights), a PowerPoint briefing on the aircraft systems, and will participate in only the standard pre-flight mission briefs.

Twenty-six class A [Ref. 3] or class A equivalent flight test accidents were surveyed to determine when the flight test accident occurred as a function of flight number. For the purpose of this survey, a class A accident is defined as a mishap that results in direct costs totaling $\$ 2,000,000$ or more, a fatality or permanent total disability, or destruction of the aircraft. As shown in Fig. 6, history shows that first flight does not have significantly more inherent risk than subsequent test flight. However, the significant disparity in training for a first flight team generally does not reflect this reality.

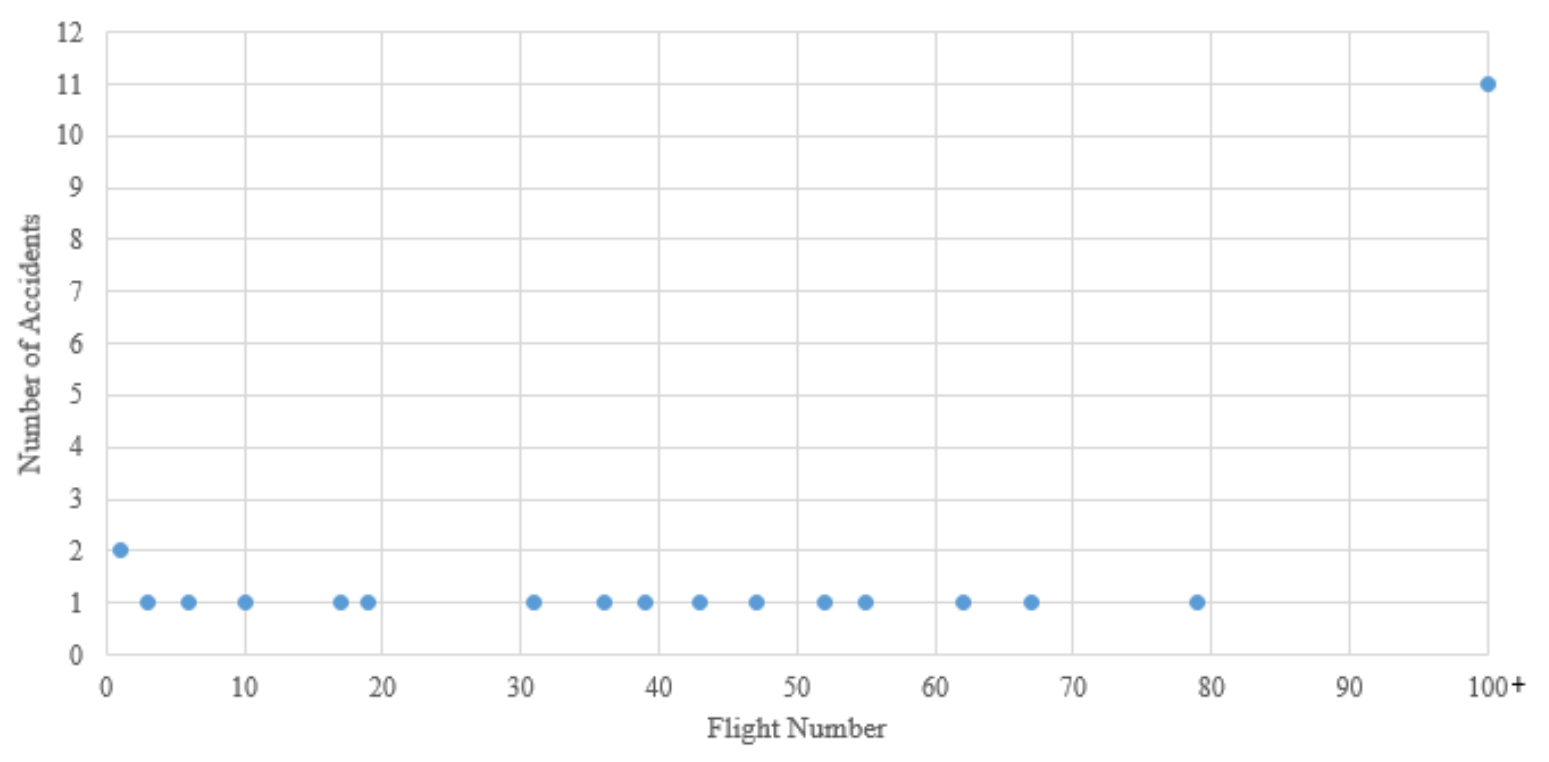

Fig. 6 Class A (or equivalent) flight test accidents as a function of flight number. Note: None of the accidents that had a structures related failure as a causal factor occurred within the first $\mathbf{4 0}$ flight. Accidents that occurred prior to 1960 or that occurred as part of a functional check flight (pre-delivery test flight) are not included in this chart.

\section{Conclusion}

Envelope expansion flight testing (specifically with respect to loads and flutter) is almost inherently considered high risk. As a result, this type of testing requires a significant investment in the education and training of the test team (to include both pilots and engineers). If implemented, the following items would almost certainly improve the learning environment and safety culture of any test team.

Although it is easy to place a cost on the implementation of the following items, it is relatively difficult to place a cost on the lack of implementation. But, this difficulty in quantifying the cost should not be confused with the idea that there is no cost.

1. Emphasize true understanding over box checking. Too often there can be a tendency to treat training plans as a box checking exercise where an expeditious completion of the plan is valued more than true knowledge. Training plans should focus on attaining true knowledge and experience.

2. Value technical expertise. Flight testing is a discipline that requires rigorous dedication to understanding not only the system under test and basic flight mechanics, but also a serious understanding of the analytical theories and background that support the type of test to be performed (e.g., loads, flutter, noise and vibration).

3. Prioritize documenting lessons learned throughout the flight test program, and develop a robust lessons learned database. A lessons learned database is an idea that generally has widespread support, but very little practical support. There is little time devoted to documenting lessons learned as a flight test program progresses. By the time a flight test program ends, there may have been significant personnel turnover and few resources left for documenting lessons learned.

4. Train for every flight as you would train for first flight. There tends to be a significant emphasis placed on training for the first flight team of a new aircraft. However, a similar level of training should be maintained 
throughout the flight test program as testing in more critical areas with less margin is performed (often by engineers with far less experience than the first flight team).

5. Look within the broader structures flight test community for training opportunities. A deficit in knowledge or experience within your local organization may be fulfilled by partnering with sister organizations that perform similar work.

6. Develop training based on individual project needs. One size fits all training does not provide sufficient depth to address specific issues and needs that arise in any given project. Additionally, any training that is not specifically applicable to a specific project for which an engineer is currently engaged will likely soon be forgotten. Direct application and practice is needed for the intended training to become part of an engineer's knowledge base.

7. Establish special qualification for high risk testing. The proficiency of both pilots and engineers should be tracked for high risk tests. Proficiency is directly related to safety.

With advances in both computing power and modeling and simulation capability, future work to incorporate highfidelity structural simulations in training and pre-flight mission simulations would represent a significant advancement in providing realistic training scenarios.

\section{References}

[1] Sorokowski, P. and Garland, M. P., "Envelope Expansion Lessons Learned,” 412TW-TIH-17-02, March 2018.

[2] Artz, B., Goodall, A., and Oswald, A. J., "If Your Boss Could Do Your Job, You're More Likely to Be Happy at Work," Harvard Business Review, published online 29 Dec. 2016. URL: https://hbr.org/2016/12/if-your-boss-could-do-your-jobyoure-more-likely-to-be-happy-at-work [retrieved 29 March 2018].

[3] Air Force Instruction 91-204, Safety Investigations and Reports, 10 April 2014. 